\title{
Optimization of Traffic Behavior via Fluid Dynamic Approach
}

\author{
Ciro D'Apice ${ }^{1}$, Rosanna Manzo ${ }^{1}$ and Benedetto Piccoli ${ }^{2}$ \\ ${ }^{1}$ Department of Information Engineering and Applied Mathematics, \\ University of Salerno, Fisciano (SA) \\ 2Istituto per le Applicazioni del Calcolo "Mauro Picone", \\ Consiglio Nazionale delle Ricerche, Roma \\ Italy
}

\section{Introduction}

The exponentially increasing number of circulating cars in modern cities renders the problem of traffic control of paramount importance. Traffic congestion is a condition on networks that occurs in the presence of an excess of vehicles on a portion of roadway, and is characterized by slower speeds, longer trip times, and increased queueing. As demand approaches the capacity of a road (or of the intersections along the road), extreme traffic congestion sets in. Incidents may cause ripple effects (a cascading failure) which then spread out and create a sustained traffic jam. The presence of hard congestions on urban networks may have dramatic implications, affecting productivity, pollution, life style, the passage of emergency vehicles traveling to their destinations where they are urgently needed. Transportation engineers and emergency planners work together to alleviate congestion and, in addition to traditional efforts, an increased focus is addressed to the development and promotion of transportation systems management and operations. The main inspiration is the understanding and optimization of traffic behavior in order to answer to several questions: where to install traffic lights or stop signs; how long the cycle of traffic lights should be; how to distribute flows at junctions, where to construct entrances, exits and overpasses, etc. in order to maximize cars flow, minimize traffic congestions, accidents, pollution.

The problem of modeling car traffic has been faced resorting to different approaches ranging from microscopic ones, taking into account each single car, to kinetic and macroscopic fluiddynamic ones, dealing with traffic situations resulting from the complex interaction of many vehicles. Each of them implies some technical approximations, and suffers therefore from related drawbacks, either analytical or computational. Here we are interested to traffic flow on a road network, modelled by a fluid-dynamic approach.

In the 1950s James Lighthill and Gerard Whitham, two experts in fluidynamics, and independently P. Richards, modeled the flow of car traffic along a single road using the same equations describing the flow of water (Lighthill et al. (1955); Richards (1956)). The basic idea is to look at large scales so as to consider cars as small particles and to assume the conservation of the cars number. The LWR model is described by a single conservation law, 
a special partial differential equation where the variable, the car density, is a conserved quantity, i.e. a quantity which can neither be created or destroyed. Then some second order models, i.e. with two equations, were proposed by Payne and Whitham (Payne $(1971 ; 1979)$; Whitham (1974)). Since the assumption of the LWR model of the dependence of the average speed $v$ only on the density $\rho$ is not valid in some situations, Payne and Whitham introduced an additional equation for the speed, including a relaxation term for $v$. Unfortunately this model suffers from sever drawbacks, which led Daganzo in 1995 to write a celebrated "requiem" for this kind of second order approximation of traffic flow (Daganzo (1995)). In particular he proved that cars may exhibit negative speed and the model violates the so-called anisotropy principle, i.e., the fact that a car should be influenced only by the traffic dynamics ahead of it, being practically insensitive to what happens behind. Finally Aw and Rascle in 2000, to overcome Daganzo's observations, proposed a "resurrection" of second order models, introducing an equation for the pressure as increasing function of the density (Aw \& Rascle (2000)). The Aw Rascle model gave origin to a lot of other traffic models and derivations. The first third order model was proposed by Helbing (see Helbing (2001)). Colombo in 2002 developed an hyperbolic phase transition model, in which the existence of the phase transition is postulated and accounted for by splitting the state space $(\rho, f)$, where $f$ is the flux, in two regions, corresponding to the regimes of free and congested flow (Colombo (2002 a;b)). A multilane extension of the Aw-Rascle model was proposed by Greenberg, Klar and Rascle (see Greenberg et al. (2003)). The idea to consider the LWR model on a network was proposed by Holden and Risebro (Holden et al. (1995)). They solved the Riemann Problem at junctions (the problem with constant initial data on each road), proposing a maximization of the flux. Existence of solution to Cauchy Problems and the counterexample to the Lipschitz continuous dependence on initial data was proved in the paper by Coclite et al. (2005). The Aw-Rascle second order model has been extended to networks in Garavello \& Piccoli (2006 b).

Traffic congestion leads to a strong degradation of the network infrastructure and accordingly reduced throughput, which can be countered via suitable control measures and strategies. Some optimization problems for road networks modeled by fluid-dynamic approach have been already studied: Helbing et al. (2005) is devoted to traffic light regulation, while Gugat et al. (2005) and Herty et al. (2003) are more related to our analysis but focus on the case of smooth solutions (not developing shocks) and boundary control. A specific traffic regulation problem is addressed in Chitour \& Piccoli (2005). Given a crossing with some expected traffic, is it preferable to construct a traffic circle or a light? The two solutions are studied in terms of flow control and the performances are compared.

In this Chapter we report some recent optimization results obtained in Cascone et al. (2007; 2008 a;b); Cutolo et al. (2009); Manzo et al. (2010) for urban traffic networks, whose evolution is described by the LWR model.

Road networks consist of a finite set of roads, that meet at some junctions. The dynamics is governed on each road by a conservation law. In order to uniquely solve the Riemann Problem at junctions and to construct solutions via Wave Front Tracking (see Bressan (2000); Garavello \& Piccoli (2006 a)), as the system is under-determined even after imposing the conservation of cars, the following assumptions are made: the incoming traffic distributes to outgoing roads according to fixed (statistical) distribution coefficients; drivers behave in order to maximize the through flux. More precisely, if the number of incoming roads is greater than that of outgoing roads, one has also to introduce right of way parameters. 
Some cost functionals have been defined to analyze the traffic behavior: $J_{1}$ measuring car average velocity, $J_{2}$ the average traveling time, $J_{3}$ the total flux of cars, $J_{4}$ the car density, $J_{5}$, the Stop and Go Waves functional (SGW), the velocity variation, $J_{6}$ the kinetic energy, and finally $J_{7}$ measuring the average traveling time weighted with the number of cars moving on each road. Notice that the cost functionals are evaluated through the use of a linear decreasing velocity function $v(\rho)=1-\rho$.

For a fixed time horizon $[0, T], \int_{0}^{T} J_{1}(t) d t, \int_{0}^{T} J_{3}(t) d t, \int_{0}^{T} J_{6}(t) d t$ have been maximized and $\int_{0}^{T}$ $J_{2}(t) d t, \int_{0}^{T} J_{7}(t) d t$ minimized, choosing as controls the right of way parameters or the distribution coefficients depending on the junctions type. A junction of $n \times m$ type is a junction with $n$ incoming roads and $m$ outgoing ones. The attention has been focused on a decentralized approach reducing the analysis of a network to simple junctions. We computed the optimal parameters for single junctions of type $1 \times 2$ and $2 \times 1$ and every initial data. For a complex network, we used the (locally) optimal parameters at every junction and we verified the performance of the (locally) optimal parameters comparing, via simulations, with other choices as fixed and random parameters. The optimization problem for junctions of $2 \times 1$ type, using as control the right of way parameter $p$, every initial data and the functionals $J_{i}, i=1,2,6,7$ (while $J_{3}$ happens to be constant) is solved in Cascone et al. (2007) and Cutolo et al. (2009). In particular the functionals $J_{2}$ and $J_{7}$ are maximized for the same values of $p$, while $J_{1}$ and $J_{6}$ have, in some cases, different optimal values. It is interesting to notice that in many cases there is a set of optimal values of the right of way parameters. Optimization results have been achieved for the functionals $J_{i}, i=1,2,3$ (see Cascone et al. (2008 a)) and $J_{6}, J_{7}$ in the case of junctions of type $1 \times 2$, using the distribution coefficient as control. Observe that the functionals $J_{6}$ and $J_{7}$ are optimized for the same values of the distribution coefficient which maximize and minimize $J_{1}$ and $J_{2}$, respectively. All the results have been tested by simulations on case studies.

Recently the problem of traffic redirection in the case an accident occurs in a congested area has been considered, see Manzo et al. (2010). Fire, police, ambulance, repair crews, emergency and life-saving equipment, services and supplies must move quickly to where the greatest need is. Assuming that emergency vehicles will cross a given incoming road $I_{\varphi r}$ $\varphi \in\{1,2\}$ and a given outgoing road $I_{\psi}, \psi \in\{3,4\}$ of a junction of type $2 \times 2$, a cost functional measuring the average velocities of such vehicles on the assigned path is analyzed. The optimization results give the values of $\alpha$ and $\beta$ (respectively, the probability that drivers go from road 1 to road 3 and from road 2 to road 3) which maximize the functional, allowing a fast transit of emergency vehicles to reach car accidents places and hospitals.

The Chapter is organized as follows. Section 2 reports the model for road networks. Riemann Solvers at junctions are described in Section 3. The subsequent Section 4 is devoted to the definition of the functionals, introduced to measure network performance. In particular in the Subsection 4.1 we optimize right of way parameters for $2 \times 1$ junctions, while in Subsection 4.1 we report optimization studies for $1 \times 2$ junctions. The Section 5 deals with some new results on the optimal redistribution of flows at nodes of type $2 \times 2$ in order to maximize the velocity of emergency vehicles on assigned paths. In all the Sections simulations are presented and discussed to illustrate the analytical optimization results.

\section{Mathematical model for road networks}

We consider a network, that is modelled by a finite set of roads $I_{k}=\left[a_{k}, b_{k}\right] \subset \mathbb{R}, k=1, \ldots, N$, $a_{k}<b_{k}$, possibly with either $a_{k}=-\infty$ or $b_{k}=+\infty$. We assume that roads are connected at 
junctions. Each junction $J$ is characterized by a finite number $n$ of incoming roads and a finite number $m$ of outgoing ones, thus we identify $J$ with $\left(\left(i_{1}, \ldots, i_{n}\right),\left(j_{1}, \ldots, j_{m}\right)\right)$. Hence, the complete model is given by a couple $(\mathcal{I}, \mathcal{J})$, where $\mathcal{I}=\left\{I_{k}: k=1, \ldots, N\right\}$ is the collection of roads and $\mathcal{J}$ is the collection of junctions. The main dependent variables introduced to describe mathematically the problem are the density of cars $\rho=\rho(t, x)$ and their average velocity $v=v(t, x)$ at time $t$ in the point $x$. From these quantities another important variable is derived, namely the flux $f=f(t, x)$ given by $f=\rho v$, which is of great interest for both theoretical and experimental purposes.

On each single road, the evolution is governed by the scalar conservation law:

$$
\partial_{t} \rho+\partial_{x} f(\rho)=0
$$

where $\rho=\rho(t, x) \in\left[0, \rho_{\max }\right],(t, x) \in \mathbb{R}^{2}$, with $\rho_{\max }$ the maximal density of cars.

The network load is described by a finite set of functions $\rho_{k}$ defined on $\left[0,+\infty\left[\times I_{k}\right.\right.$. On each road $I_{k}$ we require $\rho_{k}$ to be a weak entropic solution of the conservation law (1), that is such that for every smooth, positive function $\varphi:\left[0,+\infty\left[\times I_{k} \rightarrow \mathbb{R}\right.\right.$ with compact support on ] $0,+\infty[\times] a_{k}, b_{k}[$

$$
\int_{0}^{+\infty} \int_{a_{k}}^{b_{k}}\left(\rho_{k} \frac{\partial \varphi}{\partial t}+f\left(\rho_{k}\right) \frac{\partial \varphi}{\partial x}\right) d x d t=0,
$$

and entropy conditions are verified, see Bressan (2000); Dafermos (1999); Serre (1996).

It is well known that, for equation (1) on $\mathbb{R}$ and for every sufficiently small initial data in $B V$ (here $B V$ stands for bounded variation functions), there exists a unique weak entropic solution depending in a continuous way from the initial data in $L_{l o c}^{1}$. Moreover, for initial data in $L^{\infty} \cap L^{1}$ Lipschitz continuous dependence in $L^{1}$ is achieved.

Now we discuss how to define solutions at junctions. For this, fix a junction $J$ with $n$ incoming roads, say $I_{1}, \ldots, I_{n}$, and $m$ outgoing ones, say $I_{n+1}, \ldots, I_{n+m}$ (briefly a junction of type $n \times m$ ). A weak solution at $J$ is a collection of functions $\rho_{l}:\left[0,+\infty\left[\times I_{l} \rightarrow \mathbb{R}, l=1, \ldots, n+m\right.\right.$, such that

$$
\sum_{l=0}^{n+m}\left(\int_{0}^{+\infty} \int_{a_{l}}^{b_{l}}\left(\rho_{l} \frac{\partial \varphi_{l}}{\partial t}+f\left(\rho_{l}\right) \frac{\partial \varphi_{l}}{\partial x}\right) d x d t\right)=0,
$$

for every smooth $\varphi_{l}, l=1, \ldots, n+m$, having compact support in $\left.] 0,+\infty[\times] a_{l}, b_{l}\right]$ for $l=1, \ldots, n$ and in $] 0,+\infty\left[\times\left[a_{l}, b_{l}[\right.\right.$ for $l=n+1, \ldots, n+m$, also smooth across the junction, i.e.,

$$
\varphi_{i}\left(; b_{i}\right)=\varphi_{j}\left(; a_{j}\right), \quad \frac{\partial \varphi_{i}}{\partial x}\left(; b_{i}\right)=\frac{\partial \varphi_{j}}{\partial x}\left(; a_{j}\right), \quad i=1, \ldots, n, j=n+1, \ldots, n+m .
$$

A weak solution $\rho$ at $J$ satisfies the Rankine-Hugoniot condition at the junction, namely

$$
\sum_{i=1}^{n} f\left(\rho_{i}\left(t, b_{i}-\right)\right)=\sum_{j=n+1}^{n+m} f\left(\rho_{j}\left(t, a_{j}+\right)\right)
$$

for almost every $t>0$. This Kirchhoff type condition ensures the conservation of $\rho$ at junctions. For a system of conservation laws on the real line, a Riemann problem (RP) is a 
Cauchy Problem (CP) for an initial datum of Heavyside type, that is piecewise constant with only one discontinuity. One looks for centered solutions, i.e. $\rho(t, x)=\phi\left(\frac{x}{t}\right)$ formed by simple waves, which are the building blocks to construct solutions to the $\mathrm{CP}$ via Wave Front Tracking (WFT) algorithms. These solutions are formed by continuous waves called rarefactions and by traveling discontinuities called shocks. The speeds of the waves are related to the eigenvalues of the Jacobian matrix of $f$, see Bressan (2000). Analogously, we call RP for a junction the Cauchy Problem corresponding to initial data which are constant on each road. The discontinuity in this case is represented by the junction itself.

Definition 1 A Riemann Solver (RS) for the junction J is a map $R S: \mathbb{R}^{n} \times \mathbb{R}^{m} \rightarrow \mathbb{R}^{n} \times \mathbb{R}^{m}$ that associates to Riemann data $\rho_{0}=\left(\rho_{1,0}, \ldots, \rho_{n+m, 0}\right)$ at J a vector $\hat{\rho}=\left(\hat{\rho}_{1}, \ldots, \rho_{n+m}\right)$, so that the solution on an incoming road $I_{i}, i=1, \ldots, n$, is given by the waves produced by the $R P\left(\rho_{i}, \hat{\rho}_{i}\right)$, and on an outgoing road $I_{j}, j=n+1, \ldots, n+m$, by the waves produced by the $R P\left(\hat{\rho}_{j}, \rho_{j}\right)$. We require the consistency condition

(CC) $R S\left(R S\left(\rho_{0}\right)\right)=R S\left(\rho_{0}\right)$.

A RS is further required to guarantee the fulfillment of the following properties:

(H1) The waves generated from the junction must have negative velocities on incoming roads and positive velocities on outgoing ones.

(H2) Relation (3) holds for solutions to RPs at the junction.

(H3) The map $\rho_{0} \mapsto f(\hat{\rho})$ is continuous.

Condition (H1) is a consistency condition to well describe the dynamics at junction. In fact, if (H1) does not hold, then some waves generated by the RS disappear inside the junction. Condition (H2) is necessary to have a weak solution at the junction. However, in some cases (H2) is violated if only some components of $\rho$ have to be conserved at the junction, see for instance Garavello \& Piccoli (2006 b). Finally, (H3) is a regularity condition, necessary to have a well-posed theory. The continuity of the map $\rho_{0} \mapsto f(\hat{\rho})$ can not hold in case (H1) holds true.

There are some important consequences of property (H1), in particular some restrictions on the possible values of fluxes and densities arise. Consider, for instance, a single conservation law for a bounded quantity, e.g. $\rho \in\left[0, \rho_{\max }\right]$, and assume the following:

(F) The flux function $f:\left[0, \rho_{\max }\right] \mapsto \mathbb{R}$ is strictly concave, $f(0)=f\left(\rho_{\max }\right)=0$, thus $f$ has a unique maximum point $\sigma$.

Fixing $\rho_{\max }=1$, one example of velocity function whose corresponding flux ensures $(\mathrm{F})$ is:

Then the flux is given by

$$
v(\rho)=1-\rho .
$$

Defining:

$$
f(\rho)=\rho(1-\rho) .
$$

Definition 2 Let $\tau:\left[0, \rho_{\max }\right] \rightarrow\left[0, \rho_{\max }\right]$ be the map such that $f(\tau(\rho))=f(\rho)$ for every $\rho \in\left[0, \rho_{\max }\right]$, and $\tau(\rho) \neq \rho$ for every $\rho \in\left[0, \rho_{\max }\right] \backslash\{\sigma\}$,

we get the following:

Proposition 3 Consider a single conservation law for a bounded quantity $\rho \in\left[0, \rho_{\max }\right]$ and assume $(F)$. Let RS be a Riemann Solver for a junction, $\rho_{0}=\left(\rho_{i, 0}, \rho_{j, 0}\right)$ the initial datum and $R S\left(\rho_{0}\right)=\hat{\rho}=$ $\left(\hat{\rho}_{i}, \hat{\rho}_{j}\right)$. Then, 


$$
\begin{aligned}
& \hat{\rho}_{i} \in\left\{\begin{array}{ll}
\left.\left.\left\{\rho_{i, 0}\right\} \cup\right] \tau\left(\rho_{i, 0}\right), \rho_{\max }\right], & \text { if } 0 \leq \rho_{i, 0} \leq \sigma, \\
{\left[\sigma, \rho_{\max }\right],} & \text { if } \sigma \leq \rho_{i, 0} \leq \rho_{\max }
\end{array} \quad i=1, \ldots, n,\right. \\
& \hat{\rho}_{j} \in\left\{\begin{array}{ll}
{[0, \sigma],} & \text { if } 0 \leq \rho_{j, 0} \leq \sigma, \\
\left\{\rho_{j, 0}\right\} \cup\left[0, \tau\left(\rho_{i, 0}\right)[,\right. & \text { if } \quad \sigma \leq \rho_{j, 0} \leq \rho_{\text {max }},
\end{array} \quad j=n+1, \ldots, n+m .\right.
\end{aligned}
$$

Thanks to Proposition 3, we have the following:

Proposition 4 Consider a single conservation law for a bounded quantity $\rho \in\left[0, \rho_{\max }\right]$ and assume (F). To define a RS at a junction J, fulfilling rule (H1), it is enough to assign the flux values $f(\hat{\rho})$. Moreover, there exist maximal possible fluxes given by:

$$
\begin{gathered}
f_{i}^{\max }\left(\rho_{0}\right)=\left\{\begin{array}{ll}
f\left(\rho_{i, 0}\right), & \text { if } 0 \leq \rho_{i, 0} \leq \sigma, \\
f(\sigma), & \text { if } \sigma \leq \rho_{i, 0} \leq \rho_{\max },
\end{array} \quad i=1, \ldots, n,\right. \\
f_{j}^{\max }\left(\rho_{0}\right)=\left\{\begin{array}{ll}
f(\sigma), & \text { if } 0 \leq \rho_{j, 0} \leq \sigma, \\
f\left(\rho_{j, 0}\right), & \text { if } \sigma \leq \rho_{j, 0} \leq \rho_{\max },
\end{array} \quad j=n+1, \ldots, n+m .\right.
\end{gathered}
$$

Once a Riemann Solver $R S_{J}$ at a junction $J$ is assigned, we define admissible solutions at $J$ those $\rho$ such that $t \mapsto \rho(t, \cdot)$ is $B V$ for almost every $t$, and moreover:

where

$$
R S\left(\rho_{\text {J }}(t)\right)=\rho_{\text {I }}(t)
$$

$$
\rho_{\text {I }}(t)=\left(\rho_{1}\left(\cdot, b_{1}-\right), \ldots, \rho_{n}\left(\cdot, b_{n-}\right), \rho_{n+1}\left(\cdot, a_{n+1}+\right), \ldots, \rho_{n+m}\left(\cdot, a_{n+m}+\right)\right) .
$$

For every road $I_{k}=\left[a_{k}, b_{k}\right]$, such that either $a_{k}>-\infty$ and $I_{k}$ is not the outgoing road of any junction, or $b_{k}<+\infty$ and $I_{k}$ is not the incoming road of any junction, a boundary datum $\psi_{k}$ : $\left[0,+\infty\left[\rightarrow \mathbb{R}^{n}\right.\right.$ is given. We require $\rho_{k}$ to satisfy $\rho_{k}\left(t, a_{k}\right)=\psi_{k}(t)\left(\right.$ or $\left.\rho_{k}\left(t, b_{k}\right)=\psi_{k}(t)\right)$ in the sense of Bardos et al. (1979). For simplicity, we assume that boundary data are not necessary. The aim is to solve the $\mathrm{CP}$ for a given initial datum as in the next definition.

Definition 5 Given $\bar{\rho}_{k}: I_{k} \rightarrow \mathbb{R}^{n}, k=1, \ldots, N$, in $L_{\text {loc }}^{1}$, a collection of functions $\rho=\left(\rho_{1}, \ldots, \rho_{N}\right)$, with $\rho_{k}:\left[0,+\infty\left[\times \mathrm{I}_{k} \rightarrow \mathbb{R}^{n}\right.\right.$ continuous as function from $\left[0,+\infty\left[\right.\right.$ into $L_{\text {loc }}^{1}$, is an admissible solution to the Cauchy Problem on the network if $\rho_{k}$ is a weak entropic solution to (1) on $I_{k}, \rho_{k}(0, x)=\bar{\rho}_{k}(x)$ a.e. and at each junction $\rho$ is an admissible solution.

There is a general strategy, based on Wave Front Tracking, to prove existence of solution on a whole network for CPs.

The main steps are the following (see Garavello \& Piccoli (2006 a;b) for details):

1. Construct approximate solutions via WFT algorithms, using the $R S$ at junctions for interaction of waves with junctions.

2. Estimate the variation of flux for interaction of waves with junctions, thus on the whole network.

3. Pass to the limit using the previous steps.

In what follows we suppose that $f_{k}=f, \forall k=1, \ldots, N$, but it is possible to generalize all definitions and results to the case of different fluxes $f_{k}$ for each road $I_{k}$. In fact, all statements are in terms of values of fluxes at junctions. 


\section{Riemann Solvers according to rule (RA)}

We assume that $(\mathrm{F})$ holds true and we look for Riemman Solvers fulfilling (H1). Thus, in view of Proposition 4, it is enough to determine the fluxes values.

In Coclite et al. (2005) an RS at junctions is considered, based on the following algorithm: (RA) We assume that

(A) the traffic from incoming roads is distributed on outgoing ones according to fixed coefficients;

(B) fulfilling (A), the through flux is maximized.

Consider a junction of $n \times m$ type. For simplicity we use the notation $\gamma_{k}=f\left(\rho_{k}\right), \gamma_{k}^{\max }=f_{k}^{\max }$, $\hat{\gamma}_{k}=f\left(\hat{\rho}_{k}\right), k=1, \ldots, n+m$.

If the incoming roads are $I_{1}, \ldots, I_{n}$ and the outgoing ones $I_{n+1}, \ldots, I_{n+m}$, rule (A) corresponds to fix a stochastic matrix $A=\left(\alpha_{j, i}\right)$ where $j=n+1, \ldots, n+m$ and $i=1, \ldots, n$. The coefficient $\alpha_{j, i}$ represents the percentage of traffic from $I_{i}$ directed to $I_{j}$. Here we assume:

$$
0<\alpha_{j, i}<1, \quad \sum_{j=1}^{m} \alpha_{j, i}=1 .
$$

Recalling Proposition 4, we define:

$$
\Omega_{\text {in }}=\left[0, \gamma_{1}^{\max }\right] \times \cdots \times\left[0, \gamma_{n}^{\max }\right], \quad \Omega_{\text {out }}=\left[0, \gamma_{n+1}^{\max }\right] \times \cdots \times\left[0, \gamma_{n+m}^{\max }\right] .
$$

From (H1), one gets that the incoming fluxes must take values in $\Omega_{i n}$ and the outgoing fluxes in $\Omega_{\text {out }}$. Moreover, in order to fulfill rule (A), the incoming fluxes must belong to the region:

$$
\tilde{\Omega}_{\text {in }}=\left\{\gamma \in \Omega_{\text {in }}: A \cdot \gamma \in \Omega_{\text {out }}\right\} .
$$

Notice that $\tilde{\Omega}_{\text {in }}$ is a convex set determined by linear constraints. Moreover, rule (A) implies (H2). Thus rule (B) is equivalent to maximize only over incoming fluxes, then outgoing ones can be determined by rule (A). Finally, rules (A) and (B) correspond to a Linear Programming problem: Maximize the sum of fluxes from incoming roads over the region $\tilde{\Omega}_{i n}$. Such problem always admits a solution, which is unique provided the cost function gradient (here the vector with all components equal to 1 ) is not orthogonal to the linear constraints describing the set $\tilde{\Omega}_{i n}$.

Let us now consider a junction of type $1 \times 2$. In detail, 1 is the only incoming road, while 2 and 3 are the outgoing roads. The distribution matrix $A$ takes the form

$$
A=\left(\begin{array}{c}
\alpha \\
1-\alpha
\end{array}\right),
$$

where $\alpha \in] 0,1[$ and $1-\alpha$ indicate the percentage of cars which, from road 1, goes to road 2 and 3, respectively. Thanks to rule (B), the solution to a RP is:

$$
\hat{\gamma}=\left(\hat{\gamma}_{1}, \hat{\gamma}_{2}, \hat{\gamma}_{3}\right)=\left(\hat{\gamma}_{1}, \alpha \hat{\gamma}_{1},(1-\alpha) \hat{\gamma}_{1}\right)
$$

where 


$$
\hat{\gamma}_{1}=\min \left\{\gamma_{1}^{\max }, \frac{\gamma_{2}^{\max }}{\alpha}, \frac{\gamma_{3}^{\max }}{1-\alpha}\right\} .
$$

For a junction of $2 \times 2$ type, i.e. with two incoming roads, 1 and 2, and two outgoing roads, 3 and 4 , the traffic distribution matrix $A$ assumes the form:

$$
A=\left(\begin{array}{cc}
\alpha & \beta \\
1-\alpha & 1-\beta
\end{array}\right),
$$

where $\alpha$ is the probability that drivers go from road 1 to $\operatorname{road} 3$ and $\beta$ is the probability that drivers travel from road 2 to road 3. Let us suppose that $\alpha \neq \beta$ in order to fulfill the orthogonal condition for uniqueness of solutions. From rule (A), it follows that $\hat{\gamma}_{3}=\alpha \hat{\gamma}_{1}+$ $\beta \hat{\gamma}_{2}, \hat{\gamma}_{4}=(1-\alpha) \hat{\gamma}_{1}+(1-\beta) \hat{\gamma}_{2}$. From rule (B), we have that $\hat{\gamma}_{\varphi}, \varphi=1,2$, is found solving the Linear Programming problem:

$$
\begin{gathered}
\max \left(\gamma_{1}+\gamma_{2}\right), \\
0 \leq \gamma_{\varphi} \leq \gamma_{\varphi}^{\max }, 0 \leq \alpha \gamma_{1}+\beta \gamma_{2} \leq \gamma_{3}^{\max }, 0 \leq(1-\alpha) \gamma_{1}+(1-\beta) \gamma_{2} \leq \gamma_{4}^{\max } .
\end{gathered}
$$

The orthogonality condition can not hold if $n>m$. If not all traffic can flow to the only outgoing road, then one should assign a yielding or priority rule:

(C) There exists a priority vector $p \in \mathbb{R}^{n}$ such that the vector of incoming fluxes must be parallel to $p$.

Let us show how rule (C) works in the simple case $n=2$ and $m=1$, i.e. a junction of type $2 \times 1$. In this case, the matrix $A$ reduces to the vector $(1,1)$, thus no information is obtained. Rule (B) amounts to determining the through flux as $\Gamma=\min \left\{\gamma_{1}^{\max }+\gamma_{2}^{\max }, \gamma_{3}^{\max }\right\}$. If $\Gamma=\gamma_{1}^{\max }$ $+\gamma_{2}^{\max }$, then we simply take the maximal flux over both incoming roads. If the opposite happens, consider the space $\left(\gamma_{1}, \gamma_{2}\right)$ of possible incoming fluxes and define the following lines:

$$
\begin{gathered}
r_{p}:\{t p: t \in \mathbb{R}\}, \\
r_{\Gamma}:\left\{\left(\gamma_{1}, \gamma_{2}\right): \gamma_{1}+\gamma_{2}=\Gamma\right\} .
\end{gathered}
$$

Let $P$ be the point of intersection of the lines $r_{p}$ and $r_{\Gamma}$. Recall that the final fluxes should belong to the region:

$$
\Omega=\left\{\left(\gamma_{1}, \gamma_{2}\right): 0 \leq \gamma_{i} \leq \gamma_{i}^{\max }, i=1,2\right\} .
$$

We distinguish two cases:

a) $P$ belongs to $\Omega$;

b) $P$ is outside $\Omega$.

In the first case $\left(\hat{\gamma}_{1}, \hat{\gamma}_{2}\right)=P$, while in the second case $\left(\hat{\gamma}_{1}, \hat{\gamma}_{2}\right)=Q$, where $Q=\operatorname{proj}_{\Omega_{\Omega} r_{\Gamma}}(P)$, and proj is the usual projection on a convex set.

The reasoning can be repeated also in the case of $n$ incoming roads. In $\mathbb{R}^{n}$, the line $r_{p}$ is again given by $r_{p}=t p, t \in \mathbb{R}$, and 


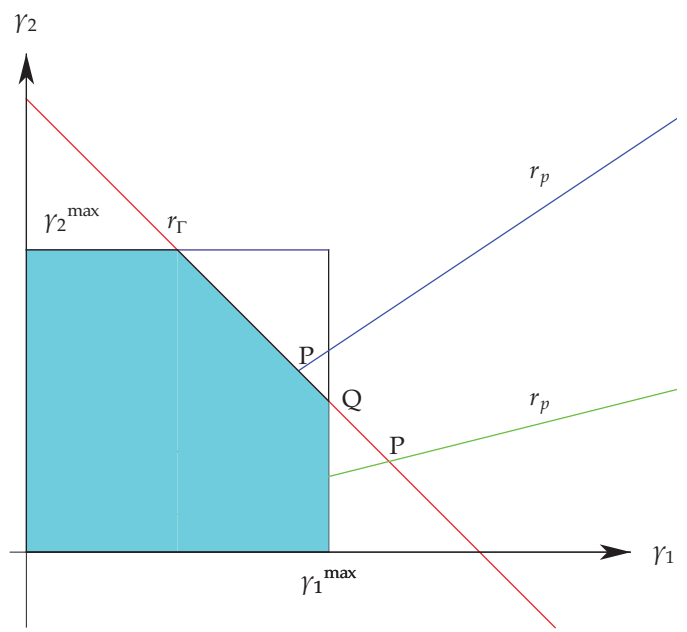

Fig. 1. The two cases: $P$ belongs to $\Omega$ and $P$ is outside $\Omega$.

$$
H_{\Gamma}=\left\{\left(\gamma_{1}, \ldots, \gamma_{n}\right): \sum_{i=1}^{n} \gamma_{i}=\Gamma\right\}
$$

is a hyperplane. There exists a unique point $P=r_{p} \cap H_{\Gamma}$. If $P \in \Omega$, then again we use $P$ to determine the incoming fluxes. Otherwise, we choose the point $Q=\operatorname{proj}_{\Omega} \cap H_{\Gamma}(P)$, the projection over the subset $\Omega \cap H_{\Gamma}$. Notice that the projection is unique since $\Omega \cap H_{\Gamma}$ is a closed convex subset of $H_{\Gamma}$. It is easy to check that (H3) is verified for this RS.

\section{Cost functionals}

We focus on a single junction with $n$ incoming roads and $m$ outgoing ones. To evaluate networks performance we define the following functionals:

$J_{1}$ measuring car average velocity:

$$
J_{1}(t)=\sum_{k=1}^{n+m} \int_{I_{k}} v\left(\rho_{k}(t, x)\right) d x,
$$

$J_{2}$ measuring average traveling time:

$$
J_{2}(t)=\sum_{k=1}^{n+m} \int_{I_{k}} \frac{1}{v\left(\rho_{k}(t, x)\right)} d x,
$$

$J_{3}$ measuring total flux of cars:

$$
J_{3}(t)=\sum_{k=1}^{n+m} \int_{I_{k}} f\left(\rho_{k}(t, x)\right) d x
$$

$J_{4}$ measuring car density: 


$$
J_{4}(t)=\sum_{k=1}^{n+m} \int_{0}^{t} \int_{I_{k}} \rho_{k}(\tau, x) d \tau d x
$$

$J_{5}$, the Stop and Go Waves functional, measuring the velocity variation:

$$
J_{5}(t)=S G W=\sum_{k=1}^{n+m} \int_{0}^{t} \int_{I_{k}}|D v(\rho)| d \tau d x,
$$

where $|D v|$ is the total variation of the distributional derivative $D \rho$, $J_{6}$ measuring the kinetic energy:

$$
J_{6}(t)=\sum_{k=1}^{n+m} \int_{I_{k}} f\left(\rho_{k}(t, x)\right) v\left(\rho_{k}(t, x)\right) d x,
$$

$J_{7}$ measuring the average traveling time weighted with the number of cars moving on each road $I_{k}$ :

$$
J_{7}(t)=\sum_{k=1}^{n+m} \int_{I_{k}} \frac{\rho_{k}(t, x)}{v\left(\rho_{k}(t, x)\right)} d x .
$$

Given a junction of type $1 \times m$ or $n \times 1$, and initial data, solving the RP we determine the average velocity, the average traveling time and the flux over the network as function of the distribution coefficients or the right of way parameters. It follows that also the functionals $J_{k}$, $k=1,2,3,6,7$ are functions of the same parameters.

For a fixed time horizon $[0, T]$, our aim is to maximize $\int_{0}^{T} J_{1}(t) d t, \int_{0}^{T} J_{3}(t) d t, \int_{0}^{T} J_{6}(t) d t$ and to minimize $\int_{0}^{T} J_{2}(t) d t, \int_{0}^{T} J_{7}(t) d t$, choosing the right of way parameters $p_{k}(t)$ or the distribution coefficients $\alpha_{k}(t)$. Since the solutions of such optimization control problems are too difficult, we reduce to the following problem:

(P) Consider a junction $J$ of $2 \times 1$ type or $1 \times 2$ type, the functionals $J_{k}, k=1,2,3,6,7$, and the right of way parameter $p_{k}(t)$ or the distribution coefficients $\alpha_{k}(t)$ as controls. We want to minimize $J_{2}(T), J_{7}(T)$ and to maximize $J_{1}(T), J_{3}(T), J_{6}(T)$ for $T$ sufficiently big. As was proved in Cascone et al. (2007; $2008 \mathrm{a} ; \mathrm{b})$, the functional $J_{3}(T)$ does not depend on the right of way parameters and on distribution coefficients.

The optimization approach we followed is of decentralized type. In fact, the optimization is done over $p$ or $\alpha$ for a single junction. For complex networks we adopt the following strategy:

Step 1. Compute the optimal parameters for single junctions and every initial data. For this, consider the asymptotic solution over the network (assuming infinite length roads so to avoid boundary data effects).

Step 2. Use the (locally) optimal parameters at every junction of the network, updating the value of the parameters at every time instant using the actual density on roads near the junction.

Step 3. Verify the performance of the (locally) optimal parameters comparing, via simulations, with other choices as fixed and random parameters.

All the optimization results reported in the following Subsections are obtained assuming the flux function (5). 


\subsection{Optimization of junctions of $2 \times 1$ type}

We focus on junctions of $2 \times 1$ type, labelling with 1 and 2 the incoming roads and with 3 the outgoing one and we consider $p$ as control (for more details see Cascone et al. (2007; 2008 b); Cutolo et al. (2009)). From the flux function we can express $\hat{\rho}_{k}$ in terms of $\hat{\gamma}_{k}$ :

$$
\hat{\rho}_{k}=\frac{1+s_{k} \sqrt{1-4 \hat{\gamma}_{k}}}{2}, k=1,2,3,
$$

with

$$
\begin{gathered}
s_{i}=\left\{\begin{aligned}
-1, \quad \text { if } \quad \rho_{i, 0}<\sigma, \gamma_{1}^{\max }+\gamma_{2}^{\max } \leq \gamma_{3}^{\max }, \\
\text { or } \quad \rho_{i, 0}<\sigma, \gamma_{3}^{\max }<\gamma_{1}^{\max }+\gamma_{2}^{\max }, p_{i} \hat{\gamma}_{3} \geq \gamma_{i}^{\max }, \quad i=1,2, \\
+1, \quad \text { if } \quad \rho_{i, 0} \geq \sigma, \\
\text { or } \quad \rho_{i, 0}<\sigma, \gamma_{3}^{\max }<\gamma_{1}^{\max }+\gamma_{2}^{\max }, p_{i} \hat{\gamma}_{3}<\gamma_{i}^{\max },
\end{aligned}\right. \\
s_{3}=\left\{\begin{aligned}
-1, \quad \text { if } \rho_{3,0} \leq \sigma, \\
\text { or } \rho_{3,0}>\sigma, \gamma_{1}^{\max }+\gamma_{2}^{\max }<\gamma_{3}^{\max }, \\
+1, \quad \text { if } \rho_{3,0}>\sigma, \gamma_{1}^{\max }+\gamma_{2}^{\max } \geq \gamma_{3}^{\max },
\end{aligned}\right.
\end{gathered}
$$

where

$$
p_{i}=\left\{\begin{array}{cc}
p, & \text { if } i=1, \\
1-p, & \text { if } i=2 .
\end{array}\right.
$$

The velocity, in terms of $\hat{\gamma}_{k}$, is given by $v\left(\hat{\rho}_{k}\right)=\frac{1-s_{k} \sqrt{1-4 \hat{\gamma}_{k}}}{2}, k=1,2,3$. The functionals $J_{2}(T)$ and $J_{7}(T)$ are maximized for the same values of $p$. In fact we get:

Theorem 6 Consider a junction J of $2 \times 1$ type. For $T$ sufficiently big, the cost functionals $J_{2}(T)$ and $J_{7}(T)$ are optimized for the following values of $p$ :

1. Case $s_{1}=s_{2}=+1$, we have that:
a. $p=\frac{1}{2}$, if $\beta^{-} \leq 1 \leq \beta^{+}$or $\gamma_{2}^{\max }=\gamma_{3}^{\max }$;
b. $p \in\left[0, p^{-}\right]$, if $\beta^{-} \leq \beta^{+} \leq 1$;
c. $p \in\left[p^{+}, 1\right]$, if $1 \leq \beta^{-} \leq \beta^{+}$;

2. Case $s_{1}=-1=-s_{2}$, we have that:
a. $\quad p=\frac{1}{2}$ or $p \in\left[p^{+}, 1\right]$, if $\beta^{-} \leq 1 \leq \beta^{+}$or $\gamma_{2}^{\max }=\gamma_{3}^{\max }$;
b. $p \in\left[0, p^{-}\right]$or $p \in\left[p^{+}, 1\right]$, if $\beta^{-} \leq \beta^{+} \leq 1$;
c. $p \in\left[p^{+}, 1\right]$, if $1 \leq \beta^{-} \leq \beta^{+}$;

3. Case $s_{1}=+1=-s_{2}$, we have that:

a. $p=\frac{1}{2}$ or $p \in\left[0, p^{-}\right]$, if $\beta^{-} \leq 1 \leq \beta^{+}$; 

b. $p \in\left[0, p^{-}\right]$, if $\beta^{-} \leq \beta^{+} \leq 1$;
c. $p \in\left[0, p^{-}\right]$or $p \in\left[p^{+}, 1\right]$, if $1 \leq \beta^{-} \leq \beta^{+}$;
d. $p=\frac{1}{2}$ or $p \in\left[p^{+}, 1\right]$, if $\gamma_{2}^{\max }=\gamma_{3}^{\max }$;

4. Case $s_{1}=s_{2}=-1$, we have that:
a. $p=\frac{1}{2}$ or $p \in\left[p^{+}, 1\right]$, if $\beta^{-} \leq 1 \leq \beta^{+}$, with $\beta^{-} \beta^{+}>1$ or $\gamma_{2}^{\max }=\gamma_{3}^{\max }$;
b. $\quad p=\frac{1}{2}$ or $p \in\left[0, p^{-}\right]$, if $\beta^{-} \leq 1 \leq \beta^{+}$, with $\beta^{-} \beta^{+}<1$;
c. $p=\frac{1}{2}$ or $p \in\left[0, p^{-}\right] \cup\left[p^{+}, 1\right]$, if $\beta^{-} \leq 1 \leq \beta^{+}$, with $\beta^{-} \beta^{+}=1$;
d. $p \in\left[0, p^{-}\right]$, if $\beta^{-} \leq \beta^{+} \leq 1$;
e. $p \in\left[p^{+}, 1\right]$, if $1 \leq \beta^{-} \leq \beta^{+}$;

where $\beta^{-}=\frac{\gamma_{3}^{\max }-\gamma_{1}^{\max }}{\gamma_{1}^{\max }}, \beta^{+}=\frac{\gamma_{2}^{\max }}{\gamma_{3}^{\max }-\gamma_{2}^{\max }}, p^{+}=\frac{\gamma_{1}^{\max }}{\gamma_{3}^{\max }}, p^{-}=\frac{\gamma_{3}^{\max }-\gamma_{2}^{\max }}{\gamma_{3}^{\max }}$.

In the particular case $\gamma_{1}^{\max }=\gamma_{2}^{\max }=\gamma_{3}^{\max }$ the functionals $J_{2}$ and $J_{7}$ are optimized for $p=\frac{1}{2}$. The maximization of the functionals $J_{1}(T)$ and $J_{6}(T)$ is reached, in some cases, for different values of the right of way parameter, as reported in the following theorem, in which the optimization analysis of the new functional $J_{6}(T)$ is compared with the results obtained in Cascone et al. (2007) for $J_{1}(T)$.

Theorem 7 Consider a junction J of $2 \times 1$ type. For $T$ sufficiently big, the cost functionals $J_{1}(T)$ and $J_{6}(T)$ are optimized for the following values of $p$ :

1. Case $s_{1}=s_{2}=+1$, we have that:
a. $p \in\left[0, p^{-}\right]$, if $\beta^{-} \leq 1 \leq \beta^{+}$, with $\beta^{-} \beta^{+}>1$, or $1 \leq \beta^{-} \leq \beta^{+}$, or $\gamma_{2}^{\max }=\gamma_{3}^{\max }$;
b. $p \in\left[0, p^{-}\right] \cup\left[p^{+}, 1\right]$, if $\beta^{-} \leq 1 \leq \beta^{+}$, with $\beta^{-} \beta^{+}=1$;
c. $p \in\left[p^{+}, 1\right]$, if $\beta^{-} \leq 1 \leq \beta^{+}$, with $\beta^{-} \beta^{+}<1$ or $\beta^{-} \leq \beta^{+} \leq 1$;

2. Case $s_{1}=-1=-s_{2}$, we have that:

for $J_{1}(T), p \in\left[p^{+}, 1\right]$;

for $J_{6}(T)$ :
a. $\quad p \in\left[p^{+}, 1\right]$, if $\beta^{-} \leq 1 \leq \beta^{+}$, or $\beta^{-} \leq \beta^{+} \leq 1$;
b. $p \in\left[0, p^{-}\right]$or $p \in\left[p^{+}, 1\right]$, if $1 \leq \beta^{-} \leq \beta^{+}$, or $\gamma_{2}^{\max }=\gamma_{3}^{\max }$;

3. Case $s_{1}=+1=-s_{2}$, we have that:

for $J_{1}(T), p \in\left[0, p^{-}\right]$;

for $J_{6}(T)$ :
a. $p \in\left[0, p^{-}\right]$, if $\beta^{-} \leq 1 \leq \beta^{+}$, or $1 \leq \beta^{-} \leq \beta^{+}$, or $\gamma_{2}^{\max }=\gamma_{3}^{\max }$;
b. $p \in\left[0, p^{-}\right]$or $p \in\left[p^{+}, 1\right]$, if $\beta^{-} \leq \beta^{+} \leq 1$;

4. Case $s_{1}=s_{2}=-1$, we have that:

for $J_{1}(T)$ : 


$$
\begin{array}{ll}
\text { a. } & p \in\left[0, p^{-}\right], \text {if } \beta^{-} \leq 1 \leq \beta^{+} \text {, with } \beta^{-} \beta^{+}<1 \text {, or } \beta^{-} \leq \beta^{+} \leq 1 \\
\text { b. } & p \in\left[0, p^{-}\right] \cup\left[p^{+}, 1\right] \text {, if } \beta^{-} \leq 1 \leq \beta^{+} \text {, with } \beta^{-} \beta^{+}=1 \\
\text { c. } & p \in\left[p^{+}, 1\right], \text { if } \beta^{-} \leq 1 \leq \beta^{+} \text {, with } \beta^{-} \beta^{+}>1 \text {, or } 1 \leq \beta^{-} \leq \beta^{+} \text {, or } \gamma_{2}^{\max }=\gamma_{3}^{\max }
\end{array}
$$

for $J_{6}(T)$ :

$$
\begin{aligned}
& \text { a. } p \in\left[0, p^{-}\right] \text {or } p \in\left[p^{+}, 1\right] \text {, if } \beta^{-} \leq 1 \leq \beta^{+} \text {, with } \beta^{-} \beta^{+}>1 \text {, or } \beta^{-} \beta^{+}<1 \text {, or } \gamma_{2}^{\max }=\gamma_{3}^{\max } \text {; } \\
& \text { b. } \quad p \in\left[0, p^{-}\right] \cup\left[p^{+}, 1\right] \text {, if } \beta^{-} \leq 1 \leq \beta^{+} \text {, with } \beta^{-} \beta^{+}=1 ; \\
& \text { c. } \quad p \in\left[p^{+}, 1\right] \text {, if } \beta^{-} \leq \beta^{+} \leq 1 \text {; } \\
& \text { d. } \quad p \in\left[0, p^{-}\right], \text {if } 1 \leq \beta^{-} \leq \beta^{+} ; \\
& \text {where } \beta^{-}=\frac{\gamma_{3}^{\max }-\gamma_{1}^{\max }}{\gamma_{1}^{\max }}, \beta^{+}=\frac{\gamma_{2}^{\max }}{\gamma_{3}^{\max }-\gamma_{2}^{\max }}, p^{+}=\frac{\gamma_{1}^{\max }}{\gamma_{3}^{\max }}, p^{-}=\frac{\gamma_{3}^{\max }-\gamma_{2}^{\max }}{\gamma_{3}^{\max }} .
\end{aligned}
$$

The functionals $J_{1}$ and $J_{6}$ are maximized for $p=0$ or $p=1$ if $\gamma_{1}^{\max }=\gamma_{2}^{\max }=\gamma_{3}^{\max }$.

The optimization algorithms are tested on Re di Roma square, a part of the urban network of Rome and on Via Parmenide crossing, a little network in Salerno (Italy).

We consider approximations obtained by the numerical method of Godunov, with space step $\Delta x=0.01$ and time step determined by the CFL condition. The road network is simulated in a time interval $[0, T]$, where $T=30 \mathrm{~min}$. As for the initial conditions on the roads of the network, we assume that, at the starting instant of simulation $(t=0)$, all roads are empty. We studied different simulation cases: right of way parameters, that optimize the cost functionals (optimal case); random right of way parameters (static random case), i.e. the right of way parameters are chosen in a random way at the beginning of the simulation process; fixed right of way parameters (fixed case), the same for each junction; dynamic random parameters (dynamic random case), i.e. right of way parameters change randomly at every step of the simulation process.

Re di Roma square is a big traffic circle with 12 roads ( 6 entering roads and 6 exiting ones), 6 junctions of $2 \times 1$ type and 6 junctions of $1 \times 2$ type. In Figure 2 (left), the topology of Re di Roma Square is reported, with junctions of $2 \times 1$ type $(1,3,5,7,9,11)$ in white, and junctions of $1 \times 2$ type $(2,4,6,8,10,12)$ in black. The traffic distribution coefficients at $1 \times 2$ junctions are determined by the road capacities (and the characteristics of the nearby portion of the Rome urban network). Therefore we focused on the right of way parameters for the $2 \times 1$ junctions, whose choice corresponds to the use of yielding and stop signs, or to the regulation of red and green phases for traffic lights.

We assume boundary conditions 0.3 for roads with non infinite endpoints and we choose for the fixed case $p=0.2$, the mean value of the static random simulations. The simulative results present some expected features and some unexpected ones. The performances of the optimal and dynamic random coefficients are definitely superior with respect to the other two. However, performances are surprisingly good, taking into account that the optimal choice was obtained by local optimization and asymptotic state, and that the dynamic random result is very close to the optimal one. Such behavior is clear for $J_{1}$ functional (see Figure 3), and even more marked for $J_{2}$ functional, which explodes for the static random and fixed parameters in case of high traffic load (see Figure 4). The explanation for such 

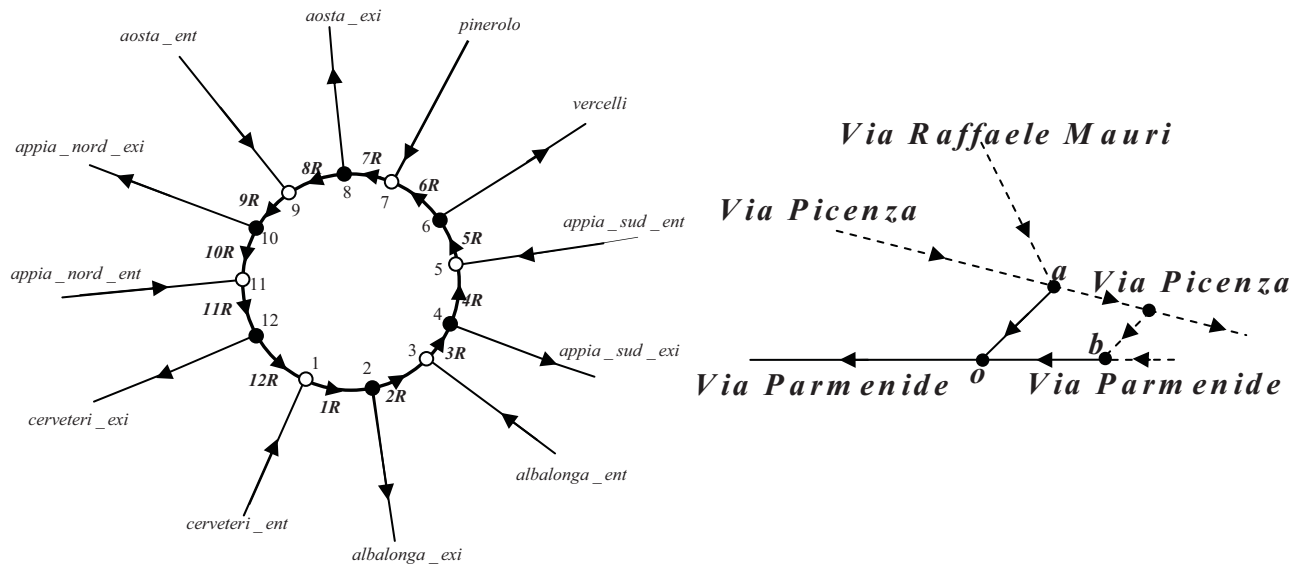

Fig. 2. Topology of Re di Roma Square (left) and Via Parmenide crossing in Salerno (right).
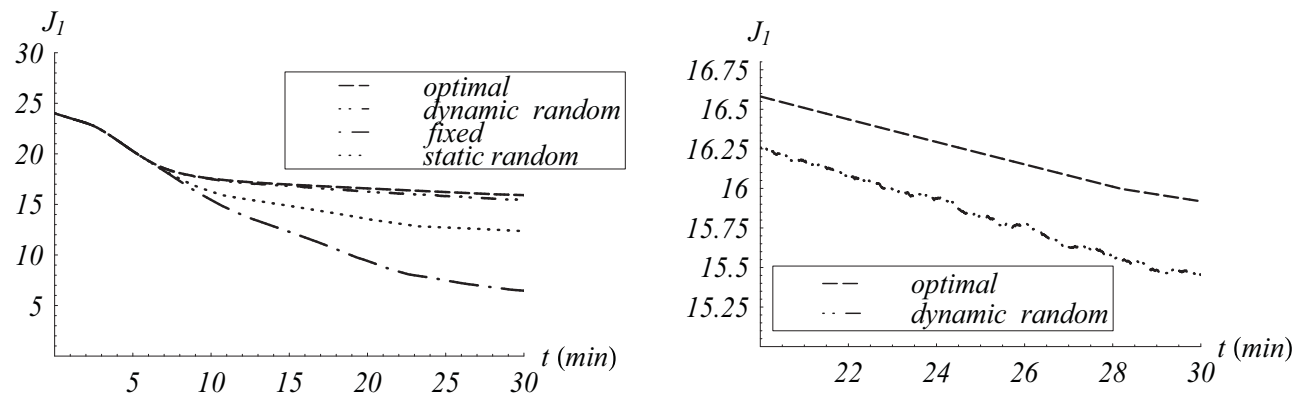

Fig. 3. $J_{1}$ simulated with zero initial conditions for all roads, boundary conditions equal to 0.3 (left) and zoom around the optimal and dynamic random case (right).
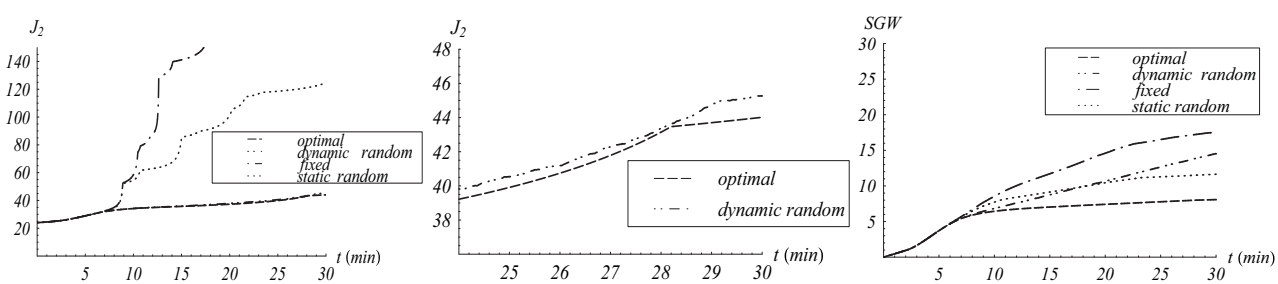

Fig. 4. $J_{2}$ simulated with zero initial conditions for all roads, boundary conditions equal to 0.3 (left) and zoom around the optimal and dynamic random case (central). Behavior of the SGW functional in the case of boundary conditions equal to 0.3 and same initial conditions for all roads (right).

explosion is the following: in some situation, the traffic circle gets completely stuck, thus the travelling time tends to infinity. This fact is also confirmed by the behavior of the cars densities on the roads, which are very irregular in the dynamic random simulations. When we consider networks with a great number of nodes, the time average of optimal parameters 
can approach 0.5 , and this justifies the similarities among dynamic random simulations and optimal ones. Hence, to discriminate between them, it is necessary to consider the SGW functional. The latter is very high for the dynamic random case and very low for the optimal one (even less than the fixed or static random case), see Figure 4 (right).

Then, we analyzed a small area of the Salerno urban network, characterized by congestion due to a traffic light, that presents a cycle with a red phase too long. In particular, in Figure 2 (right) the portion of the interested area is depicted. We focus on the crossing indicated by $o$. The incoming road from point $a$ to point $o$ (a portion of Via Mauri, that we call $\operatorname{road} a-o$ ) is very short and connects Via Picenza to Via Parmenide. The incoming road from point $b$ to point $o$ (that we call road $b-o$ ) is a part of Via Parmenide. Crossing $o$ is ruled by a traffic light, with a cycle of 120 seconds, where the phase of green is 15 seconds for drivers, who travel on the road $a-o$. It is evident that such situation leads to very high traffic densities on the road $a-0$ as the little duration of green phase does not always allow to absorb queues. From a probabilistic point of view, we can say that road $b-o$ has a right of way parameter, that is: $p=\frac{105}{120}=0.875$, while road $a-o$ has a right of way parameter $q=1-p=0.125$.

This particular crossing has been studied in order to understand how to improve the conditions of traffic in presence of a traffic light. We considered a boundary data 0.8 for roads, that enter the junction $o$ and a boundary condition 0.3 for the outgoing road. Figure 5 (left) reports the functional $J_{1}$ in optimal, dynamic random and fixed cases. It is evident that the optimal case is higher than the fixed simulation (that corresponds to the real case $p=$ 0.875); hence, actually, Via Parmenide in Salerno does not follow a traffic optimization policy. In fact there are some time intervals in which cars are stopped by the traffic light, while other roads are completely empty. This situation means that the cycle of the traffic light is too long. A solution could be to reduce the cycle or substitute the traffic light with a stop sign.

Simulations show that dynamic random algorithms and optimization approaches are totally different for Via Parmenide, respect to Re di Roma square. This is due to the nature itself of the dynamic random simulation (Figure 5, right), that is similar to a fixed case with $p=0.5$, which is the minimum for $J_{1}$. For Via Parmenide, only one traffic parameter is used, whose analytical optimization gives a solution far from 0.5 ; hence, the dynamic random simulation and optimal ones cannot be similar.
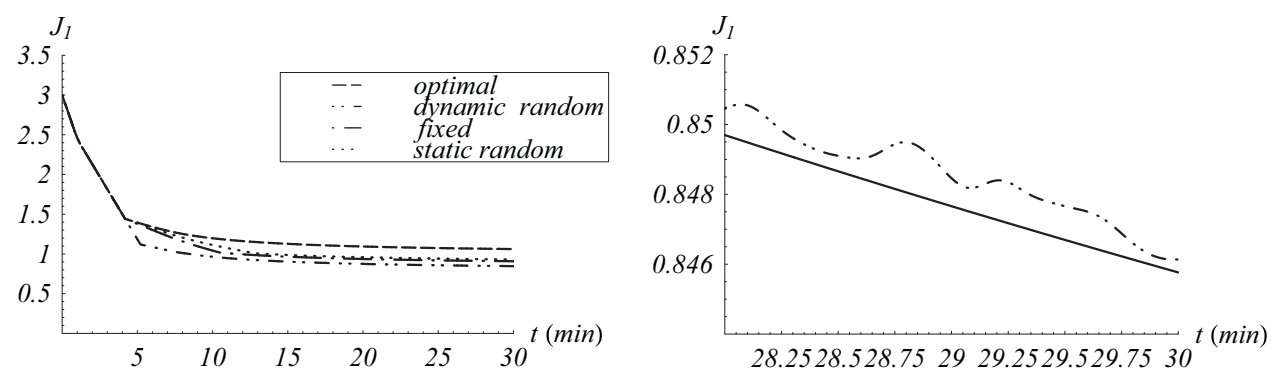

Fig. 5. Left: behavior of $J_{1}$ for Via Parmenide crossing. Right: behavior of $J_{1}$ for Via Parmenide crossing among the dynamic random simulation (dot dot dashed line) and the fixed simulation with $p=0.5$ (solid line). 
The optimizations of local type, like the ones that we are considering here, could not necessarily imply global performance improvements for big networks. Table 1 reports the distribution coefficients used for simulations of Re di Roma Square. Roads cerveteri_exi, albalonga_exi, appia_sud_exi, vercelli, aosta_exi, appia_nord_exi have a boundary data equal to 0.4 , while the other ones equal to 0.35 .

\begin{tabular}{|c|c|c|}
\hline Junction $i$ & $\alpha_{i R,(i-1) R}$ & $\alpha_{i,(i-1) R}$ \\
\hline$i=2$ & 0.866071 & 0.133929 \\
\hline$i=4$ & 0.459854 & 0.540146 \\
\hline$i=6$ & 0.800971 & 0.199029 \\
\hline$i=8$ & 0.730612 & 0.269388 \\
\hline$i=10$ & 0.536050 & 0.463950 \\
\hline$i=12$ & 0.753927 & 0.246073 \\
\hline
\end{tabular}

Table 1. Traffic distribution parameters for junctions of $1 \times 2$ type in Re di Roma Square.

We show that for the chosen initial data the algorithm for the maximization of velocity assures globally the best performance for the network, also in terms of average times, and kinetic energy (see Figures 6 and 7). The goodness of opt $J_{1}$ for global performances is confirmed by the behavior of $J_{2}$. In fact, opt $J_{2} J_{7}$ and opt $J_{6}$ can let $J_{2}$ explode, i.e. the traffic circle is stuck and the time to run inside goes to infinity. This situation is more evident in the total kinetic energy, $J_{6}$, which tends to zero when opt $J_{1}$ is not used. This means that the cars flux is going to zero, as evident from Figure 8 (left), hence roads inside the circle are becoming full. A consequence of this phenomenon is also visible in $J_{7}$ evolution, that tends to infinity.
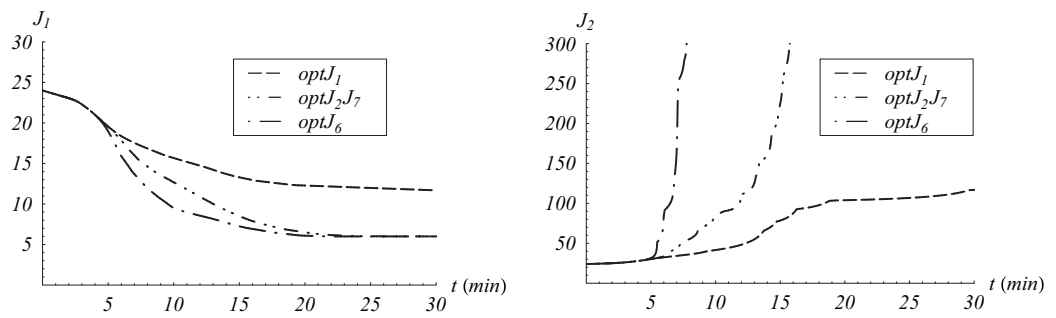

Fig. 6. Behavior of $J_{1}$ (left) and $J_{2}$ (right), using the parameter $p$ which optimizes $J_{1}$, opt $J_{1}, J_{2}$ and $J_{7}, \mathrm{opt} J_{2} J_{7}$, and $J_{6}$, opt $J_{6}$.
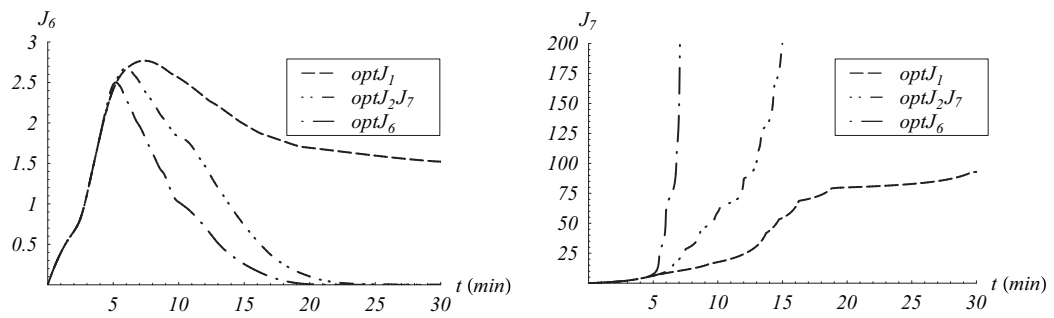

Fig. 7. Behavior of $J_{6}$ (left) and $J_{7}$ (right), using the parameter $p$ which optimizes $J_{1}$, opt $J_{1}, J_{2}$ and $J_{7}$, opt $J_{2} J_{7}$, and $J_{6}$, opt $J_{6}$. 

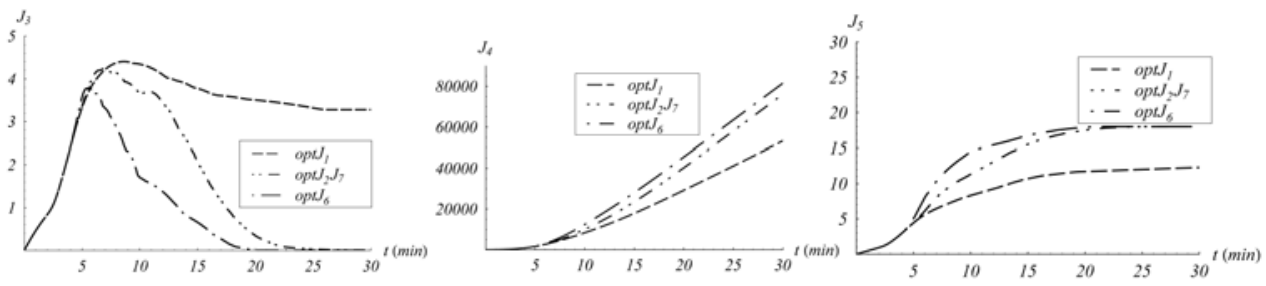

Fig. 8. Behavior of the optimal cost functionals $J_{3}$ (left), $J_{4}$ (central) and $S G W$ (right).

Observe that the amount of traffic load, visible in $J_{4}$ (Figure 8 (central)), tends to decrease using the priority parameter which maximizes $J_{1}$. Moreover, the behavior of $J_{5}$ (Figure 8 (right)), that measures the velocity variation, indicates that the use of opt $J_{1}$ leads to more regular densities on roads, giving advantages in terms of security. Remember that there are no optimization algorithms for the functionals $J_{k}, k=3,4,5$ and they are computed directly using opt $J_{1}$, opt $J_{2} J_{7}$ and opt $J_{6}$.

\subsection{Optimization of junctions of $1 \times 2$ type}

We focus on junctions of $1 \times 2$ type, labelling with 1 the incoming road and with 2 and 3 the outgoing ones and we consider $\alpha$ as control. For more details, see Cascone et al. (2008 a).

Theorem 8 Consider a junction J of $1 \times 2$ type and $T$ sufficiently big. The cost functionals $J_{1}(T)$, $J_{2}(T), J_{6}(T)$ and $J_{7}(T)$ are optimized for $\alpha=\frac{1}{2}$, with the exception of the following cases (in some of them, the optimal control does not exist but is approximated ):

1. if $\gamma_{2}^{\max }<\gamma_{1}^{\max } \leq \gamma_{3}^{\max }$ and $\gamma_{2}^{\max }<\frac{\gamma_{1}^{\max }}{2}, \alpha=\alpha_{2}$;

2. if $\gamma_{2}^{\max } \leq \gamma_{3}^{\max } \leq \gamma_{1}^{\max }$ and $\gamma_{3}^{\max } \leq \frac{\gamma_{1}^{\max }}{2}, \alpha=\alpha_{1}+\varepsilon$;

3. if $\gamma_{2}^{\max } \leq \gamma_{3}^{\max } \leq \gamma_{1}^{\max }, \alpha=\frac{\gamma_{2}^{\max }}{\gamma_{2}^{\max }+\gamma_{3}^{\max }}$;

4. if $\gamma_{3}^{\max } \leq \gamma_{2}^{\max }<\gamma_{1}^{\max }$, we have to distinguish three cases:

- if $\alpha_{2}=\frac{1}{2}, \alpha=\frac{1}{2}-\varepsilon$;

- if $\frac{1}{2} \leq \alpha_{1}<\alpha_{2}, \alpha=\alpha_{1}+\varepsilon$;

- if $\alpha_{1}<\alpha_{2} \leq \frac{1}{2}, \alpha=\alpha_{2}-\varepsilon$;

where $\alpha_{1}=1-\frac{\gamma_{3}^{\max }}{\gamma_{1}^{\max }}, \alpha_{2}=\frac{\gamma_{2}^{\max }}{\gamma_{1}^{\max }}$ and $\varepsilon$ is small and positive.

We present simulation results for a road network, that consists of 6 junctions of $1 \times 2$ and $2 \times 2$ type, see Figure 9.

For every junction of $2 \times 2$ type, we set all the entries of the distribution matrix $A$ equal to 0.5. Hence, no control is considered for such junctions. The network is simulated in such conditions: initial data equal to 0.3 for all roads at the starting instant of simulation $(t=0)$; boundary data of Dirichlet type, equal to 0.45 for road $a_{1}$, while for roads $a_{3}, c_{2}, f_{1}$, and $f_{2}$, we 


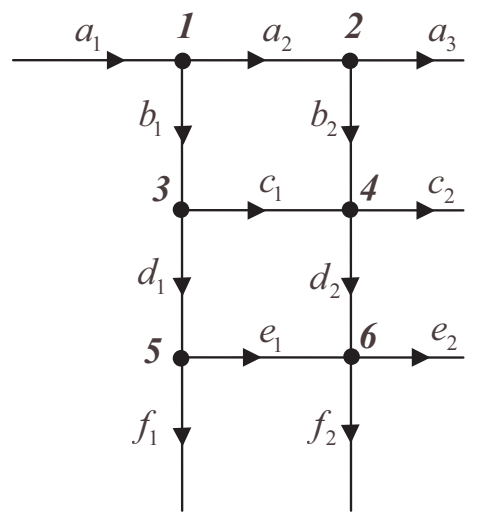

Fig. 9. Topology of the network.
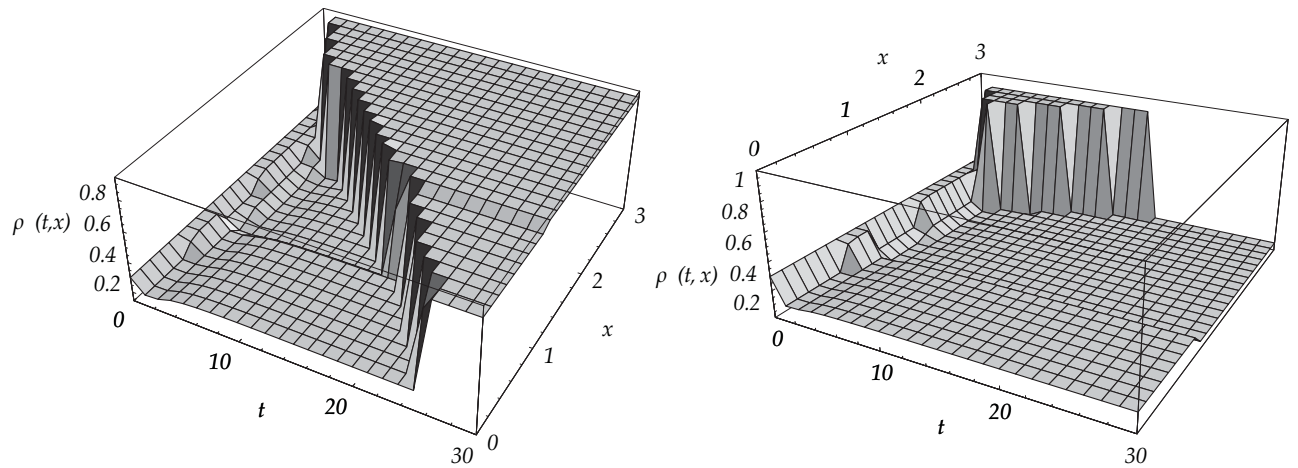

Fig. 10. Left. Density $\rho(t, x)$ on roads $b_{1}$ (from 0 to 1 on the axis $x$ ), $d_{1}$ (from 1 to 2 on the axis $x$ ) and $f_{1}$ (from 2 to 3 on the axis $x$ ), with $\alpha=0.2$ for $1 \times 2$ junctions. Right. Density $\rho(t, x)$ on roads $b_{1}$ (from 0 to 1 on the axis $x$ ), $d_{1}$ (from 1 to 2 on the axis $x$ ) and $f_{1}$ (from 2 to 3 on the axis $x$ ), with optimal distribution coefficients for $1 \times 2$ junctions.

choose a Dirichlet boundary data equal to 0.9 ; time interval of simulation $[0, T]$, where $T=30 \mathrm{~min}$. We analyzed traffic conditions for different values of $\alpha$. Figure 10 (left) reports the density $\rho(t, x)$ on roads $b_{1}, d_{1}$ and $f_{1}$ for $\alpha=0.2$, assuming that all roads have length equal to 1 . High levels of density interest these vertical roads, hence they tend to be more heavily congested than others. This can be seen in Figure 10 where, at $t=10$, the road $f_{1}$ is already congested with a density value almost equal to 0.9 . At $t=25$, the intense traffic of roads $f_{1}$ propagates backward and influences roads $b_{1}$ and $d_{1}$. The traffic densities on other roads is very low.

When we deal with the optimal choice of the distribution coefficients, densities on roads $c_{2}$, $e_{2}, f_{1}$ and $f_{2}$ tend to increase. However, the optimal choice better redistributes traffic flows on the whole network, as we can see from Figure 10 (right), that shows the car density $\rho(t, x)$ for roads $b_{1}, d_{1}$ and $f_{1}$.

Then, we compared three scenarios $(\alpha=0.2, \alpha=0.8$ and optimal $\alpha)$. We concluded that a real decongestion effect is evident for optimal distribution coefficients (see Figure 11, that 
shows the cost functionals $J_{1}$ and $J_{2}$ ). This phenomenon is also evident for the behaviors of $J_{6}$ and $J_{7}$, see Figure 12. In fact, the kinetic energy, represented by $J_{6}$, tends to zero when congestion problems are evident, as in the case $\alpha=0.2$. This means that the cars flux is going to zero and roads of the network are becoming to be full. An improvement of car traffic is obtained for $\alpha=0.8$ but the better situation in terms of viability is always reached in the optimal case. Indeed, for the cost functional $J_{7}$, the presence of decongestion phenomena is more evident when networks parameters are not the optimal ones. In fact, fixing $\alpha=0.2$ and $\alpha=0.8, J_{7}$ tends to infinity for big times, meaning that the velocity of cars is decreasing, with consequent filling of roads.

The dynamic random simulation follows the behavior of the optimal one, as we can see from Figure 13 (left). One could ask if an optimization is necessary, since random choices leads to similar functional values. The dynamic random simulation, in the reality of urban networks, implies that drivers flow is very chaotic, since drivers choices rapidly change during their own travel. Let us show this phenomenon considering the Stop and Go Waves functional $(S G W)$.

Figure 13 (right) shows a great variation of velocity for the dynamic random choice, which implies a higher probability of car accidents. Note that the optimal case for $S G W$ is simulated according to the optimization algorithm for the cost functionals $J_{1}$ and $J_{2}$ (and not for SGW itself).

From a statistical point of view, it is possible to understand why dynamic random simulations are very similar to the optimal case for functionals $J_{1}$ and $J_{2}$. From Theorem 8 , the optimal choice for the distribution coefficient is almost always 0.5, and this is the expected average value of random choices.
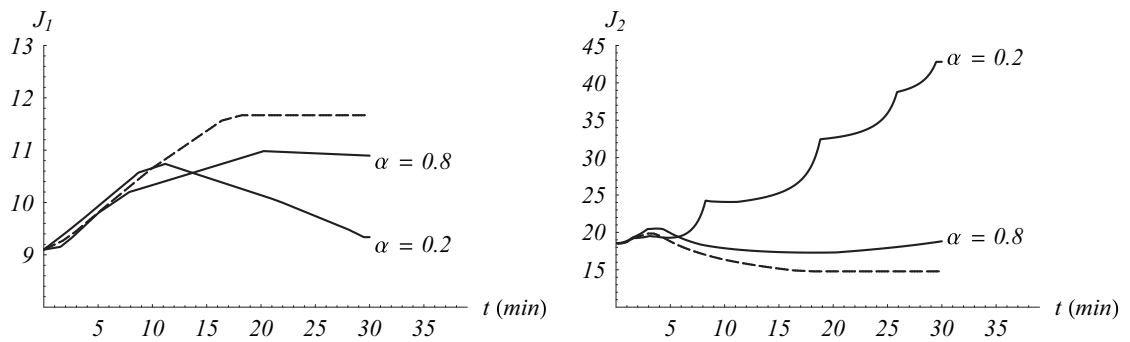

Fig. 11. $J_{1}$ (left) and $J_{2}$ (right). Solid lines: fixed cases for different values of the distribution coefficient; dashed line: optimal simulation.
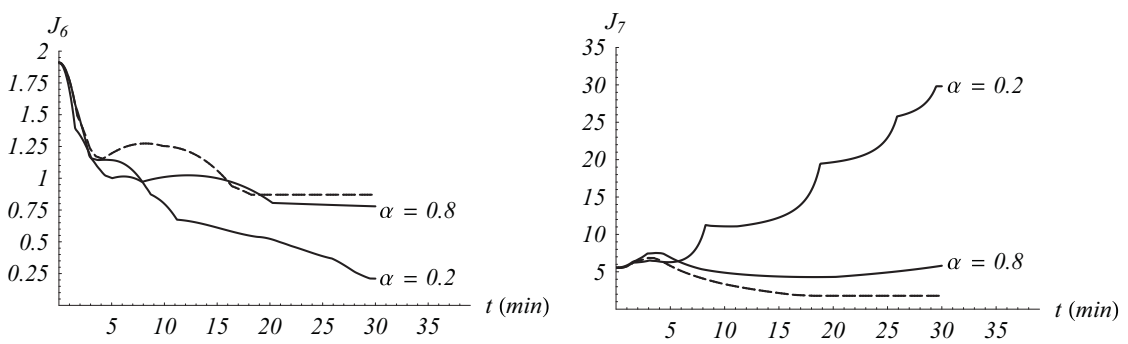

Fig. 12. $J_{6}$ (left) and $J_{7}$ (right). Solid lines: fixed cases for different values of the distribution coefficient; dashed line: optimal simulation. 

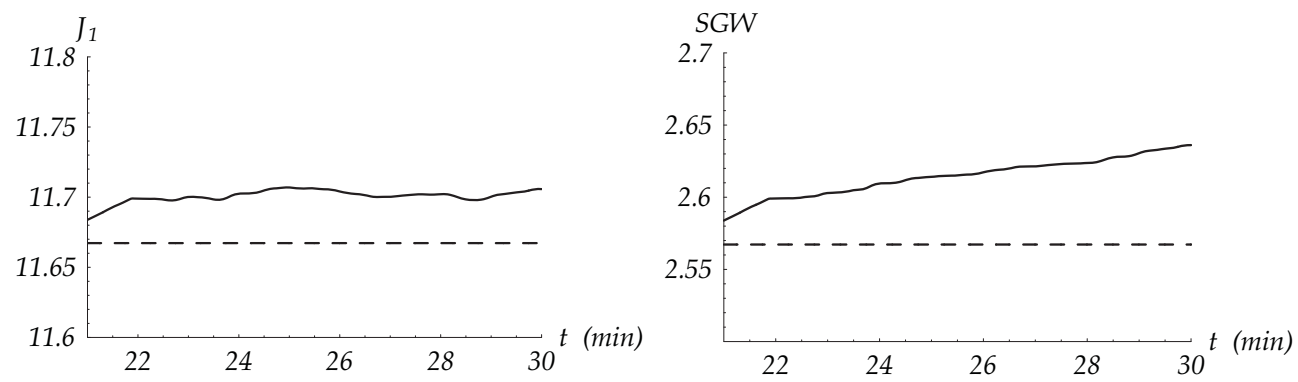

Fig. 13. Left: comparison among the dynamic random simulation and the optimal case for $J_{1}$. Right: behavior of $S G W$ in the optimal and in the dynamic random case; dashed line: optimal simulation.

\section{Optimal distribution of traffic flows at junctions in emergency cases}

The problem we face here is to find the values of traffic distribution parameters at a junction in order to manage critical situations, such as car accidents. In this case, beside the ordinary cars flows, other traffic sources, due to emergency vehicles, are present. More precisely, assume that a car accident occurs on a road of an urban network and that some emergency vehicles have to reach the position of the accident, or of a hospital.

We define a velocity function for such vehicles:

$$
\omega(\rho)=1-\delta+\delta v(\rho)
$$

with $0<\delta<1$ and $v(\rho)$ as in (4). Since $\omega\left(\rho_{\max }\right)=1-\delta>0$, it follows that the emergency vehicles travel with a higher velocity with respect to cars. Notice that (6) refers to the previous formula coincides with the velocity of the ordinary traffic for $\delta=1$.

Consider a junction $J$ with 2 incoming roads and 2 outgoing ones. Fix an incoming road $I_{\varphi r}$ $\varphi=1,2$, and an outgoing road $I_{\psi}, \psi=3,4$. Given an initial data $\left(\rho_{\varphi, 0}, \rho_{\psi, 0}\right)$, we define the cost functional $W_{\varphi, \varphi}(t)$, which indicates the average velocity of emergency vehicles crossing $I_{\varphi}$ and $I_{\psi}$ :

$$
W_{\varphi, \psi}(t)=\int_{I_{\varphi}} \omega\left(\rho_{\varphi}(t, x)\right) d x+\int_{I_{\psi}} \omega\left(\rho_{\psi}(t, x)\right) d x .
$$

For a fixed time horizon $[0, T]$, the aim is to maximize $\int_{0}^{T} W_{\varphi, \psi}(t)$ by a suitable choice of the traffic distribution parameters $\alpha_{\psi, \varphi}$ for $T$ sufficiently big.

Assigned the path consisting of roads 1 and 3, the cost functional $W_{1,3}(T)$ is optimized choosing the distribution coefficients according to the following theorem (for more details see Manzo et al. (2010)).

Theorem 9 For a junction J of $2 \times 2$ type and $T$ sufficiently big, the cost functional $W_{1,3}(T)$ is maximized for $\alpha=1-\frac{\gamma_{4}^{\max }}{\gamma_{1}^{\max }}, 0 \leq \beta<1-\frac{\gamma_{4}^{\max }}{\gamma_{1}^{\max }}$, with the exception of the following cases, where the optimal controls do not exist but are approximated by:

- $\alpha=\varepsilon_{1}, \beta=\varepsilon_{2}$, if $\gamma_{1}^{\max } \leq \gamma_{4}^{\max }$; 


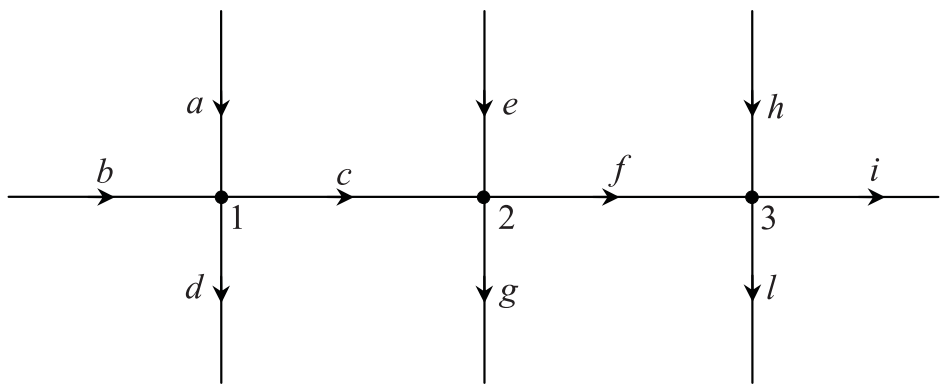

Fig. 14. Topology of the cascade junction network.

- $\quad \alpha=\frac{\gamma_{3}^{\max }}{\gamma_{3}^{\max }+\gamma_{4}^{\max }}-\varepsilon_{1}, \beta=\frac{\gamma_{3}^{\max }}{\gamma_{3}^{\max }+\gamma_{4}^{\max }}-\varepsilon_{2}$, if $\gamma_{1}^{\max }>\gamma_{3}^{\max }+\gamma_{4}^{\max }$,

for $\varepsilon_{1}$ and $\varepsilon_{2}$ small, positive and such that $\varepsilon_{1} \neq \varepsilon_{2}$.

Consider the network in Figure 14, described by 10 roads, divided into two subsets, $R_{1}=\{a, d, e, g, h, l\}$ and $R_{2}=\{b, c, f, i\}$ that are, respectively, the set of inner and external roads. Assuming that the emergency vehicles have an assigned path, we analyze the behavior of the functional:

$$
W(t)=W_{a c}(t)+W_{e f}(t)+W_{h i}(t)
$$

The evolution of traffic flows is simulated using the Godunov scheme with $\Delta x=0.0125$, and $\Delta t=\frac{\Delta x}{2}$ in a time interval $[0, T]$, where $T=100 \mathrm{~min}$. Initial and boundary data are chosen in order to simulate a network with critical conditions on some roads, as congestions due to the presence of accidents (see Table 2).

\begin{tabular}{|c|c|c|}
\hline Road & Initial condition & Boundary data \\
\hline$a$ & 0.1 & 0.1 \\
\hline$b$ & 0.65 & 0.65 \\
\hline$c$ & 0.75 & $/$ \\
\hline$d$ & 0.95 & 0.95 \\
\hline$e$ & 0.2 & 0.2 \\
\hline$f$ & 0.65 & $/$ \\
\hline$g$ & 0.95 & 0.95 \\
\hline$h$ & 0.25 & 0.25 \\
\hline$i$ & 0.55 & 0.55 \\
\hline$l$ & 0.95 & 0.95 \\
\hline
\end{tabular}

Table 2. Initial conditions and boundary data for roads of the cascade junction network.

Figure 15 shows the temporal behavior of $W(t)$ measured on the whole network. As we can see, the optimal cost functional is higher than the random ones, hence the principal aim is achieved for the chosen data set. Notice that, in general, optimal global performances on networks could also not be achieved, as the traffic state is strictly dependent on initial and boundary data. 

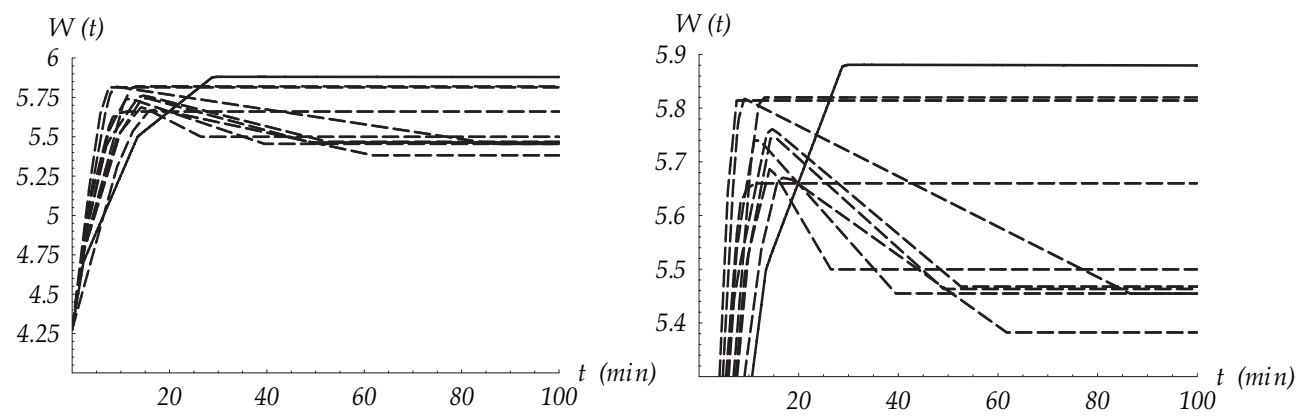

Fig. 15. Evolution of $W(t)$ for optimal choices (continuous line) and random parameters (dashed line); left: behavior in [0,T]; right: zoom around the asymptotic values.

\section{Conclusions}

Traffic regulation techniques for the optimization of car traffic flows in congested urban networks was considered. The approach used for the description of traffic flows is of fluiddynamic type. The main advantages of this approach, with respect to existing ones, can be summarized as follows. The fluid-dynamic models are completely evolutive, thus they are able to describe the traffic situation of a network at every instant of time. This overcomes the difficulties encountered by many static models. An accurate description of queues formation and evolution on the network is possible. The theory permits the development of efficient numerical schemes also for very large networks. This is possible since traffic at junctions is modelled in a simple and computationally convenient way (resorting to a linear programming problem). The performance analysis of the networks was made through the use of different cost functionals, measuring car average velocity weighted or not weighted with the number of cars moving on each road, the average travelling time, velocity variation, kinetic energy, etc. Exact analytical results were given for simple junctions of $1 \times 2$ and $2 \times 1$ type, and then used in order to simulate more complex urban networks. Moreover the problem of emergency vehicles transit has been treated. The problem has been faced choosing a route for emergency vehicles (not dedicated, i.e. not limited only to emergency needs) and redistributing traffic flows at junctions on the basis of the current traffic load in such way that emergency vehicles could travel at the maximum allowed speed along the assigned roads (and without blocking the traffic on other roads). All the optimization results have been obtained using a decentralized approach, i.e. an approach which sets local optimal parameters for each junction of the network. In future we aim to extend the optimization results to more general junctions and to explore global optimization techniques. In addition, the definition and optimization of functionals which take into account the emission and propagation of pollutants produced by cars might provide powerful technological tools to rationalize the design and use of public and private transportation resources, and to reduce unpleasant effects of urban traffic on the environment. 


\section{References}

Aw, A. \& Rascle, M. (2000). Resurrection of "second order" models of traffic flow?, SIAM J. Appl. Math., Vol. 60, pp. 916-938.

Bardos, C.; Le Roux, A.Y. \& Nedelec, J.C. (1979). First Order Quasilinear Equations with Boundary Conditions, Commun. Partial Differential Equations, Vol. 4, pp. 1017-1034.

Bressan, A. (2000). Hyperbolic Systems of Conservation Laws - The One-dimensional Cauchy Problem, Oxford Univ. Press.

Cascone, A.; D'Apice, C.; Piccoli, B. \& Rarità, L. (2007). Optimization of traffic on road networks, Mathematical Models and Methods in Applied Sciences, Vol. 17, No. 10, pp. 1587-1617.

Cascone, A.; D'Apice, C; Piccoli, B. \& Rarità, L. (2008). Circulation of car traffic in congested urban areas, Communication in Mathematical Sciences, Vol. 6, No. 3, pp. 765-784.

Cascone A.; Manzo, R.; Piccoli B. \& Rarità L. (2008). Optimal vs randomness for car traffic regulation, Physical Review E, Statistical, Nonlinear, And Soft Matter Physics, Vol. 78, No. 2, 026113(5).

Chitour Y. \& Piccoli B. (2005). Traffic circles and timing of traffic lights for cars flow, Discrete and Continous Dynamical Systems-Series B, Vol. 5, pp. 599-630.

Coclite, G.M.; Garavello, M. \& Piccoli, B. (2005). Traffic flow on a road network, SIAM J. Math. Anal., Vol. 36, No. 6, pp. 1862-1886.

Colombo, R.M. (2002). Hyperbolic phase transitions in traffic flow, SIAM J. Appl. Math., Vol. 63, No. 2, pp. 708-721.

Colombo, R.M. (2002). On a 2x2 hyperbolic traffic flow model, Math. Comput. Modelling, Vol. 35, pp. 683-688.

Cutolo, A.; D'Apice, C. \& Manzo, R. (2009). Traffic optimization at junctions to improve vehicular flows, Preprint D.I.I.M.A., No. 57.

Dafermos, C. (1999). Hyperbolic Conservation Laws in Continuum Physics, Springer-Verlag.

Daganzo, C. (1995). Requiem for second-order fluid approximation to traffic flow, Transportation Res., Part B, Vol. 29, pp. 277-286.

Garavello, M. \& Piccoli, B. (2006). Traffic Flow on Networks, Applied Math Series Vol. 1, American Institute of Mathematical Sciences.

Garavello, M. \& Piccoli, B. (2006). Traffic flow on a road network using the Aw-Rascle model, Commun. Partial Differential Equations, Vol. 31, No. 2, pp. 243-275.

Greenberg, J.M.; Klar, A. \& Rascle, M. (2003). Congestion on multilane highways, SIAM J. Appl. Math., Vol. 63, No. 3, pp. 818-833.

Gugat, M.; Herty, M.; Klar A. \& Leugering, G. (2005). Optimal Control for Traffic Flow Networks, Journal of Optimization Theory and Applications, Vol. 126, pp. 589-616.

Helbing, D. (2001). Traffic and related self-driven many-particle systems, Reviews of Modern Physics, Vol. 73, pp. 1067-1141.

Helbing, D.; Lammer, S. \& Lebacque, J. P. (2005). Self-organized control of irregular or perturbed network traffic, Optimal Control and Dynamic Games, C. Deissenberg and R.F. Hartl eds., Springer, Dordrecht, pp. 239-274.

Herty, M. \& Klar, A. (2003). Modelling, Simulation and Optimization of Traffic Flow Networks, SIAM J. Sci. Comp., Vol. 25, pp. 1066-1087.

Holden, H. \& Risebro N.H. (1995). A mathematical model of traffic flow on a network of unidirectional roads, SIAM J. Math. Anal., Vol. 26, pp. 999-1017. 
Lighthill, M.J. \& Whitham, G.B. (1955). On kinetic waves. II. Theory of traffic flows on long crowded roads, Proc. Roy. Soc. London Ser. A, Vol. 229, pp. 317-345.

Manzo, R.; Piccoli, B. \& Rarità, L. (2010). Optimal distribution of traffic flows at junctions in emergency cases, European Journal on Applied Mathematics, submitted.

Payne, H.J. (1971). Models of freeway traffic and control, Simulation Council Proc., Vol. 1, pp. 51-61.

Payne, H.J. (1979). FREFLO: a macroscopic simulation model of freeway traffic, Transportation Research Record, Vol. 722, pp. 68-75.

Richards, P.I. (1956). Shock waves on the highway, Oper. Res., Vol. 4, pp. 42-51.

Serre, D. (1996). Systems of conservation laws I and II, Diderot Editeur, Paris.

Whitham, G.B. (1974). Linear and Nonlinear Waves, Pure and Applied Math., WileyInterscience, New York. 


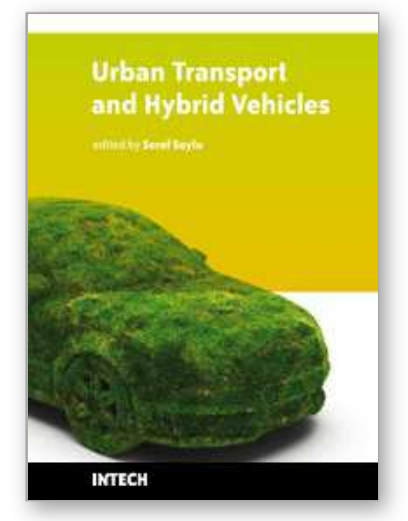

\section{Urban Transport and Hybrid Vehicles}

Edited by Seref Soylu

ISBN 978-953-307-100-8

Hard cover, 192 pages

Publisher Sciyo

Published online 18, August, 2010

Published in print edition August, 2010

This book is the result of valuable contributions from many researchers who work on both technical and nontechnical sides of the field to be remedy for typical road transport problems. Many research results are merged together to make this book a guide for industry, academia and policy makers.

\section{How to reference}

In order to correctly reference this scholarly work, feel free to copy and paste the following:

Benedetto Piccoli, Ciro D'Apice and Rosanna Manzo (2010). Optimization of Traffic Behavior via Fluid Dynamic Approach, Urban Transport and Hybrid Vehicles, Seref Soylu (Ed.), ISBN: 978-953-307-100-8, InTech, Available from: http://www.intechopen.com/books/urban-transport-and-hybrid-vehicles/optimization-of-trafficbehavior-via-fluid-dynamic-approach

\section{INTECH}

open science | open minds

\section{InTech Europe}

University Campus STeP Ri

Slavka Krautzeka 83/A

51000 Rijeka, Croatia

Phone: +385 (51) 770447

Fax: +385 (51) 686166

www.intechopen.com

\section{InTech China}

Unit 405, Office Block, Hotel Equatorial Shanghai

No.65, Yan An Road (West), Shanghai, 200040, China

中国上海市延安西路65号上海国际贵都大饭店办公楼405单元

Phone: +86-21-62489820

Fax: +86-21-62489821 
(C) 2010 The Author(s). Licensee IntechOpen. This chapter is distributed under the terms of the Creative Commons Attribution-NonCommercialShareAlike-3.0 License, which permits use, distribution and reproduction for non-commercial purposes, provided the original is properly cited and derivative works building on this content are distributed under the same license. 